\title{
A Robust Zero-Watermark Copyright Protection Scheme Based on DWT and Image Normalization
}

\author{
Mahsa Shakeri and Mansour Jamzad \\ Computer Engineering Department, \\ Sharif University of Technology, Tehran, Iran \\ mshakeri@ce.sharif.edu \\ Jamzadesharif.edu
}

\begin{abstract}
Recently, protecting the copyright of digital media has become an imperative issue due to the growing illegal reproduction and modification of digital media. A large number of digital watermarking algorithms have been proposed to protect the integrity and copyright of images. Traditional watermarking schemes protect image copyright by embedding a watermark in the spatial or frequency domain of an image. However, these methods degrade the quality of the original image in some extend. In recent years, a new approach called zero-watermarking algorithms is introduced. In these methods, the watermark does not require to be embedded into the protected image but is used to generate a verification map which is registered to a trusted authority for further protection. In this paper a robust copyright proving scheme based on discrete wavelet transform is proposed. It uses a normalization procedure to provide robustness against geometric distortions and a cellular automaton for noise robustness. Experimental results on images with different complexity demonstrate that our proposed scheme is robust against common geometric and non geometric attacks including blurring, JPEG compression, noise addition, sharpening, scaling, rotation, and cropping. In addition, our experimental results obtained on images with different complexities showed that our method could outperform the related methods in most cases.
\end{abstract}

Keywords: Zero-watermarking, copyright protection, discrete wavelet transform, image normalization, cellular automata, robustness.

\section{$1 \quad$ Introduction}

With the rapid expansion of Multimedia and the networking technology, the problem of illegal reproduction and modification of digital media has become increasingly important. The watermarking technique is one of the solutions for image copyright protection. Traditional watermarking methods [1, 2, 3], embed a watermark logo in the spatial or frequency domain of an original image so that the watermark information can be extracted for copyright protection. However, this embedding procedure degrades the original image's quality. Therefore, a new watermarking approach called zero-watermarking has been proposed in which the quality of the original image does not change, but the watermark is constructed by extracting features of the original 
image. Recently, a group of zero-watermarking methods have been introduced [4, 5, $6,8,9]$ that extract some binary features from the host image. Then by applying exclusive-or between these extracted features and the binary logo, a verification map is achieved. This verification map is registered in a trusted authority for ownership protection. In this paper, we propose a novel scheme from this group of zerowatermarking methods.

In [4], Chen et al. proposed a wavelet-based copyright proving scheme in which the features of host image were extracted from t-level LL subband. This scheme is not robust enough under some geometric attacks. A new wavelet-based zero-watermarking algorithm was proposed in [5] that achieved better robustness than [4] by applying a kind of smoothing filter on t-level LL subband. An adaptive approach proposed in [6] which allowed image owners to adjust the strength of watermarks through a threshold by using Sobel technology [7]. In [8] genetic algorithm is used to provide a proper threshold. As these two schemes depend on the edge features that are extracted from an image, the wrong threshold can increase false positive significantly. A copyright protection method for digital image with $1 / \mathrm{T}$ rate forward error correction was proposed in [9]. The watermark logo is fused with noise bits to improve the security and later became exclusive-or with the feature value of the image by $1 / \mathrm{T}$ rate forward error correction. Although this method has high performance on non geometric attacks, it still does not achieve high performance for geometric attacks such as rotation and shearing. In this paper, we use discrete wavelet transform and a normalization procedure to extract binary features and compare our scheme with [4], [5] and [9] schemes.

The rest of this paper is organized as follows: In section 2 discrete wavelet transform, cellular automata and the procedure of image normalization is presented. The proposed method is elaborated in section 3 followed by the simulation results and performance analysis in section 4. Finally, the conclusions are summed up in section 5.

\section{Related Knowledge}

\subsection{Discrete Wavelet Transformation (DWT)}

The discrete wavelet transformation is a mathematical tool for decomposing functions which can examine an image in the time and frequency domains, simultaneously. The transformed image is obtained by repeatedly filtering the image on a row-by-row and column-by-column basis. After each level wavelet transformation, the low frequency subband which contains most of the energy in the image may be transformed again. If the process is repeated times, it is called t-level wavelet transformation and the low frequency subband is referred by $L L_{t}$. As the low frequency components in DWT are less sensitive under signal processing attacks than the high frequency components, we used the subband $L L_{t}$ of the original image in our proposed method.

\subsection{Cellular Automaton}

A bi-dimensional cellular automaton is a triple $A=(S, N \delta)$; where $\mathrm{S}$ is a nonempty set, called the state set, $N \subseteq \mathbb{Z}^{2}$ is the neighborhood, and $\delta: S^{N} \rightarrow S$ is the local tran- 
sition function (rule); the argument of $\delta$ indicates the states of the neighborhood cells at a given time, while its value represents the central cell state at the next time.

A digital image is a bi-dimensional array of $n \times n$ pixels. Therefore, in this paper our model is based on a bi-dimensional symmetric cellular automata of the form $A=(S, N \delta)$ with $S=\{\#, 0,1, \ldots, k-1\}$ for an image with $k$ gray levels. \# is the quiescent state associated to the cells outside the grid. $\mathrm{N}$ is the Moor neighborhood (the eight neighboring cells surrounding a cell) while the transition function $\delta: S^{9} \rightarrow$ $S$ is defined as follows:

$$
\delta\left(\left(s_{i}\right)_{i=1}^{9}\right)=\left\{\begin{aligned}
\sum_{i=1}^{9} s_{i} / 9, & \text { if } s_{5} \neq \# \\
\#, & \text { if } s_{5}=\#
\end{aligned}\right.
$$

A cell (not being in the quiescent state) changes its state to the mean state of the cells in the neighborhood. The cells disposed outside the lattice of $n \times n$ pixels are assumed to be in the quiescent.[10]

\subsection{Affine Transform}

An image $g(x, y)$ is said to be an affine transform of $f(x, y)$ if there is a matrix $A=$ $\left(\begin{array}{ll}a_{11} & a_{12} \\ a_{21} & a_{22}\end{array}\right)$ and vector $d=\left(\begin{array}{l}d_{1} \\ d_{2}\end{array}\right)$ such that $g(x, y)=f\left(x_{a}, y_{a}\right)$, where

$$
\left(\begin{array}{l}
x_{\mathrm{a}} \\
y_{\mathrm{a}}
\end{array}\right)=\mathrm{A} \cdot\left(\begin{array}{l}
\mathrm{x} \\
\mathrm{y}
\end{array}\right)-\mathrm{d}
$$

It can be seen that geometric transformation such as: rotation, scaling, translation, shearing are all the special cases of affine transform.

Lemma 1: If $g(x, y)$ is an affine transformed image of $f(x, y)$ obtained with affine matrix $A=\left(\begin{array}{ll}a_{11} & a_{12} \\ a_{21} & a_{22}\end{array}\right)$ and $d=0$, then the following identities hold:

$$
\begin{aligned}
& m_{p q}^{\prime}=\sum_{i=0}^{p} \sum_{j=0}^{q}\left(\begin{array}{c}
p \\
i
\end{array}\right)\left(\begin{array}{c}
q \\
j
\end{array}\right) a_{11}^{i} \cdot a_{12}^{p-i} \cdot a_{21}^{j} \cdot a_{22}^{q-j} \cdot m_{i+j, p+q-i-j} \\
& \mu_{p q}^{\prime}=\sum_{i=0}^{p} \sum_{j=0}^{q}\left(\begin{array}{c}
p \\
i
\end{array}\right)\left(\begin{array}{c}
q \\
j
\end{array}\right) a_{11}^{i} \cdot a_{12}^{p-i} \cdot a_{21}^{j} \cdot a_{22}^{q-j} \cdot \mu_{i+j, p+q-i-j} .
\end{aligned}
$$

where $\mathrm{m}_{\mathrm{pq}}^{\prime}, \mu_{\mathrm{pq}}^{\prime}$ are the moments of $g(x, y)$, and $\mathrm{m}_{\mathrm{pq}}, \mathrm{m}_{\mathrm{pq}}$ are the moments of $f(x, y)$.

\subsection{Image Normalization}

The key idea of the proposed watermarking scheme is to extract binary features from a normalized image in both watermark embedding and extraction phases. For image normalization, five different affine transforms are applied to the original image $f(x, y)$ by which the geometric distortions of the image are eliminated [11,12]. The normalization procedure for a given image $f(x, y)$ consists of five steps. Let $m_{p q}$ and $\mu_{p q}, p, q=0,1,2, \ldots$ be the geometric and central moments of image, respectively. The normalization steps are as follows: 
Step 1: The translation is eliminated by setting the center of the image $f(x, y)$ at point $\left(\begin{array}{l}d_{1} \\ d_{2}\end{array}\right)$ with $d_{1}=\frac{m_{10}}{m_{00}}$ and $d_{2}=\frac{m_{01}}{m_{00}}$ in (2). $m_{00}, m_{01}$ and $m_{10}$ are the moments of $f(x, y)$. The resulting image is denoted by $f_{1}(x, y)[11]$.

Step 2: Apply a shearing transform to $f_{1}(x, y)$ in the $\mathrm{x}$ direction with matrix $\mathrm{A}=\left(\begin{array}{ll}1 & \beta \\ 0 & 1\end{array}\right)$ and $d=0$ in (2). The parameter $\beta$ is set in a way that the resulting image, denoted by $f_{2}(x, y)$ achieves $\mu_{30}^{(2)}=0$ [11], where the superscript is used to denote $f_{2}(x, y)$. According to equation (4), we have

$$
\mu_{30}^{(2)}=\mu_{30}^{(1)}+3 \beta \mu_{21}^{(1)}+3 \beta^{2} \mu_{12}^{(1)}+\beta^{3} \mu_{03}^{(1)} .
$$

where $\mu_{p q}^{(1)}$ are the central moments of $f_{1}(x, y)$.

Step 3: Apply a shearing transform to $f_{2}(x, y)$ in the y direction with matrix $A=$ $\left(\begin{array}{ll}1 & 0 \\ \gamma & 1\end{array}\right)$ and $d=0$. The parameter $\gamma$ is set in a way that the resulting image, denoted by $f_{3}(x, y)$ achieves $\mu_{11}^{(3)}=0$ [11]. From identity (4), we have

$$
\mu_{11}^{(3)}=\gamma \mu_{20}^{(2)}+\mu_{11}^{(2)} \text {. }
$$

Step 4: Scale $f_{3}(x, y)$ in both $\mathrm{x}$ and y directions with $A=\left(\begin{array}{ll}\alpha & 0 \\ 0 & \delta\end{array}\right)$ and $d=0$. The parameters $\alpha$ and $\delta$ are determined in a way that the resulting image denoted by $f_{4}(x, y)$ achieves a determined standard size and its moments become $\mu_{50}^{(4)}>0$ and $\mu_{05}^{(4)}>0$ [11].

Step 5: Apply a rotation transform to $f_{4}(x, y)$ with matrix $A=\left(\begin{array}{cc}\cos \emptyset & \sin \emptyset \\ -\sin \emptyset & \cos \emptyset\end{array}\right)$ and $d=0$. The parameter $\emptyset$ is determined in a way that the resulting image denoted by $f_{5}(x, y)$ achieves $\mu_{03}^{(5)}+\mu_{21}^{(5)}<0$ [12]. The image $f_{5}(x, y)$ is the normalized image that is used in our watermarking method.

Theorem 1: An image $f(x, y)$ and its affine transform have the same normalized image.

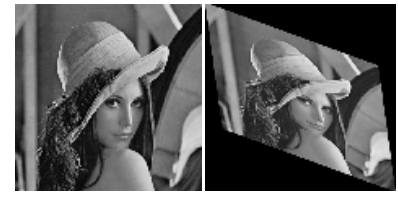

(a) (b)

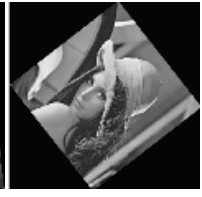

(c)

Fig. 1. (a) Original image Lena. (b) The Lena image after shearing distortion in $x$ and $y$ directions. (c) Normalized image from both (a) and (b). 
This theorem has been proved in [11]. Fig. 1 illustrates the normalization procedure. The original image "Lena" is shown in Fig. 1(a). Also, this image after affine distortion is shown in Fig. 1(b). Both of these images yield the same normalized image, illustrated in Fig. 1 (c), when the above normalization procedure is applied.

In our proposed scheme, we first obtain the normalized image and then extract binary features so that our method becomes robust against geometric distortions because according to Theorem 1 and as shown in Fig. 1, an image and its affine transform (image with geometric distortions) have the same normalized image.

\section{The Proposed Method}

A zero-watermarking method consists of two phases: the signature extraction procedure and the watermark verification procedure. In the first phase some binary features are extracted from the original image. Then bitwise exclusive-or is applied on the binary map image and a permuted binary logo to generate the verification map. Finally, the verification map that is a kind of signature is sent to a trusted authority. The second phase consists of retrieving the binary logo and authenticating the copyright of the image. Fig. 2 shows the steps of the signature extraction procedure and Fig. 3 illustrates the verification procedure. The detailed description of these two phases is stated in sections 3.1 and 3.2.

\subsection{Signature Extraction Procedure}

Let the original image $O$ be a gray level image of size $H_{O} \times W_{O}$ and the digital logo $W$ be a binary image of size $H_{w} \times W_{w}$. The original image $O$ and the binary logo $W$ are defined as follows:

$$
\begin{aligned}
& O=\left\{o_{i j} \mid 0 \leq o_{i j} \leq 255,0 \leq i \leq H_{O}, 0 \leq j \leq W_{O}\right\} . \\
& W=\left\{w_{i j}, w_{i j} \in\{0,1\}, 0 \leq i \leq H_{w}, 0 \leq j \leq W_{w}\right\} .
\end{aligned}
$$

Step 1) Choosing the appropriate level of wavelet transform: Assuming the original image and the binary logo are of size $2^{n}$, the original image will be decomposed by using $t$ level wavelet transformation in step 3 to obtain the low frequency subband $L L_{t}$. The value of $\mathrm{t}$ is determined by the following formula:

$$
2^{t}=\frac{W_{O}}{W_{w}}=\frac{H_{O}}{H_{w}} .
$$

Step 2) Normalization: The normalization procedure which has been described in section 2.4 is applied on the original image to obtain the normalized image.

Step 3) Discrete wavelet transformation of the normalized image: The normalized image is decomposed by using $t$ level wavelet transformation to obtain the low frequency subband $L L_{t}$. Let call it $\mathrm{L}$ and define it as follows:

$$
L=\left\{L_{i j} \mid 0 \leq L_{i j} \leq 255,0 \leq i \leq H_{L}, 0 \leq j \leq W_{L}\right\} .
$$

where $W_{L}$ and $H_{L}$ are the width and height of $L$. 


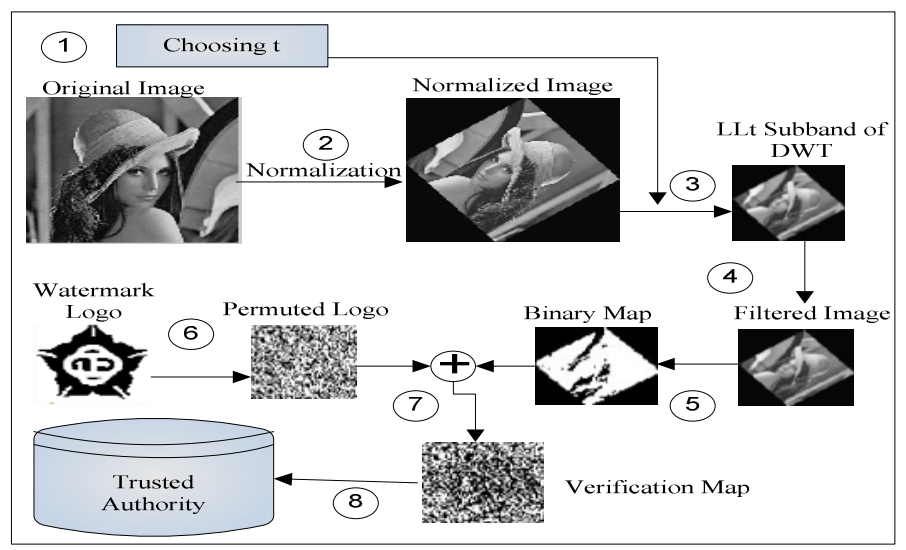

Fig. 2. The digital signature extraction procedure of our proposed method

Step 4) Cellular Automata on L: A two-dimensional cellular automata is used as described in section 2.2 for noise filtering over each pixel of the image L. The resulted image is called LS.

Step 5) Binary map generation: Let the gray level avg be taken as the average value of the image LS. Then the binary map $p$ is generated as follows:

$$
p_{i j}=\left\{\begin{array}{l}
0, \text { if } \operatorname{LS}(i, j)<a v g \\
1, \text { if } \operatorname{LS}(i, j) \geq a v g
\end{array}\right.
$$

Step 6) Logo permutation: To increase randomness, the logo $\mathrm{W}$ is scrambled by a two dimensional pseudorandom permutation with seed s [13]. The permuted logo is denoted by $W$.

$$
\dot{W}=\left\{\dot{w}_{i j} \mid \dot{w}_{i j}=P R P_{s}\left(w_{i j}\right), 0 \leq i, i \leq H_{w}, 0 \leq j, j \leq W_{w}\right\}
$$

where $P R P_{s}$ is the permuted function with seed $s$.

Step 7) Verification map generation: We apply bitwise exclusive-or between the binary map $p$ and the permuted logo $W$ to generate the verification map $\mathrm{K}$ as follows:

$$
K=P \oplus \dot{\mathrm{W}} \text {. }
$$

Step 8) Digital signature and timestamping: The resulted verification map $K$, the width and height of the original image, the seed $s$, the value $t$ and the signer's identity $I D_{\text {signer }}$ is sent to a trusted authority. The trusted authority center generates the certificate $C_{T A}$ for the host image as follows:

$$
C_{T A}=\operatorname{Hash}_{T A}\left(K\left\|W_{O}\right\| \mathrm{H}_{\mathrm{O}}\left\|I D_{\text {signer }}\right\| s\|t\| \mathrm{ts}\right) .
$$

where $\operatorname{Hash}_{T A}($.$) is the one-way hash function, t s$ is a timestamp and II is the concatenation operator. At last, the trusted authority center publishes the certificate $C_{T A}$, timestamp $t s$ and the hash function $\operatorname{Hash}_{T A}($.$) on its bulletin board.$ 


\subsection{Watermark Verification Procedure}

Step 1) Getting the information from trusted authority: First, the verifier receives the $C_{T A}$, timestamp $t s$ and hash function $\operatorname{Hash}_{T A}($.$) from trusted authority. Then C_{T A}{ }^{*}$ is computed by using $\left\{K, W_{O}, \mathrm{H}_{0}, I D_{\text {signer }}, s, t\right\}$ and $t s$ as follows:

$$
C_{T A}{ }^{*}=\operatorname{Hash}_{T A}\left(K\left\|W_{O}\right\| \mathrm{H}_{\mathrm{O}}\left\|I D_{\text {signer }}\right\| s\|t\| \mathrm{ts}\right) .
$$

If the resulted $C_{T A}{ }^{*}$ will not be the same as $C_{T A}$ the verification procedure is terminated in this phase; otherwise, the validity of the information is proven.

Step 2) Normalization: Normalize the test image by applying the indicated normalization procedure.

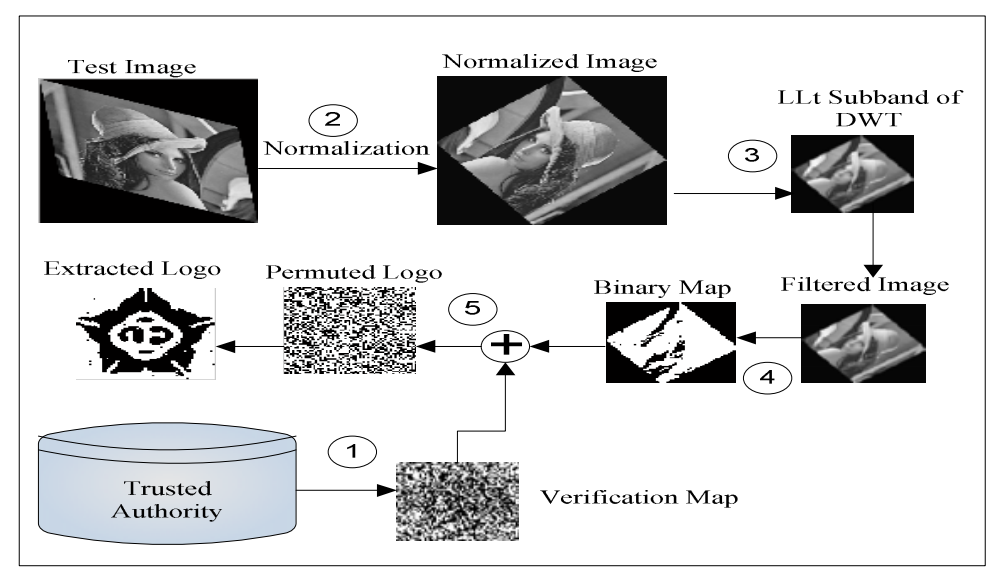

Fig. 3. Watermark verification procedure of our proposed method

Step 3) Discrete wavelet transformation and filtering by Cellular automata: The normalized test image is decomposed by performing t-level wavelet transform to obtain the subband $L L_{t}$. Then the cellular automata as described in section 2.2 is applied to achieve ĹS.

Step 4) Binary map generation: The binary map $p$ is generated by calculating the average value of L'S as noted in signing procedure.

Step 5) Retrieving the logo: By applying exclusive-or between the binary map $p$ and the verification map $K$ the permuted logo $\tilde{W}$ is obtained as follows:

$$
\tilde{W}=\dot{p} \oplus \mathrm{K} .
$$

The inverse pseudorandom permutation of the permuted logo $\tilde{W}$ is computed by function $P R P_{s}^{-1}$ and seed $s$. the retrieved $\log 0 \widetilde{W}$ is as follows: 


$$
\widetilde{W}=\left\{\widetilde{w}_{i j} \mid \widetilde{w}_{i j}=P R P_{s}^{-1}\left(\widetilde{w}_{l j}\right), 0 \leq i, i \leq H_{w}, 0 \leq j, j \leq W_{w}\right\} .
$$

Finally, the verifier can visually verify the accuracy of retrieved logo and validate the ownership of the test image.

\section{Experimental Results and Analysis}

We conducted some experiments to demonstrate the robustness of our copyright proving scheme against signal processing and geometric attacks. In addition, we compared our method with other related works.

\subsection{Experimental Results}

We measure the quality between the original image and the attacked image by using the peak signal to noise ratio (PSNR) which is defined as follows:

$$
P S N R=10 \log _{10} \frac{\max \left(O_{i j}\right)^{2} \times W_{O} \times H_{O}}{\sum\left(O_{i j}-O_{l \jmath}^{\prime}\right)^{2}} .
$$

where $O$ and $O$ are the original image and the attacked image, respectively. $W_{O}$ is the width and $H_{O}$ is the height of image $O$.

After extracting the watermark the normalized cross correlation (NC) between the original watermark and the extracted logo is computed to evaluate the correctness of the extracted logo. The NC formula for binary watermark is defined as follows:

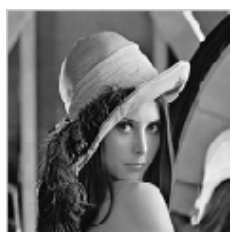

(a)

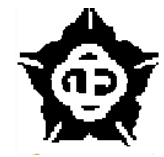

(b)

Fig. 4. (a)Test image 'Lena'. (b) Binary watermark logo.

$$
N C\left(w, w^{\prime}\right)=\frac{\sum_{i=1}^{H_{w}} \sum_{j=1}^{W_{w}} w_{i j} \times w_{l j}^{\prime}}{\sum_{i=1}^{H_{W}} \sum_{j=1}^{W_{w}} w_{i j}{ }^{2}} .
$$

$w_{i j}$ and $w_{l j}$ are the values that are located in the coordinate (i,j) of the original watermark $w$ and the extracted watermark logo $w$. Here $w_{i j}$ is set to -1 if it is equal to 0 . Otherwise, it is set to 1 . The bits of $w_{l j}^{\prime}$ is set in the same way. Therefore, the value of $\mathrm{NC}$ will be in the range of $[-11]$. 


\subsection{Applying Attacks to the Original Image}

In this paper we used the same original image and binary logo as [4] and [5] for better comparison. Fig. 4 shows the original 256 gray-level Lena image of size $512 \times 512$ and the binary logo of size $64 \times 64$.

Attack in zero-watermarking methods is defined as any kind of distortions that can be applied on the original image in order to disturb the procedure of binary feature extraction in watermark verification phase. Different signal processing and geometric attacks were applied on the test image and the Normalized cross correlation between the retrieved logo and the original logo is computed. In addition the related methods [4], [5] and [9] have been implemented and the results of our proposed method are compared with them. Following is the list of attacks that were applied to the test image.

Attack 1. Gaussian blurring with two pixels radius.

Attack 2. JPEG compression with compression rate $13 \%$.

Attack 3. Sharpening the test image.

Attack 4. 7\% Gaussian noise .

Attack 5. Reducing the size to $128 \times 128$ then resizing it to $512 \times 512$

Attack 6 . $10^{\circ}$ rotation followed by resizing to $512 \times 512$.

Attack 7. 250 pixel quarter cropping and a 60 pixel surrounded cropping.

Attack 8. 2 unit shearing transform in $\mathrm{x}$ and $\mathrm{y}$ directions.

Attack 9. The blind pattern matching attack (BPM) [4].

Tables 1 and 2 show the NC of the proposed method for Lena under the abovementioned nongeometric and geometric attacks. As shown in these two Tables the average NC between the retrieved watermark and the original logo is 0.96. Also, according to the retrieved watermark logos in Table 1 and 2, the proposed scheme can retrieve clear and recognizable digital logos from the attacked images.

Moreover, the average NC of [4], [5], [9] and the proposed scheme for different test images are computed. Unlike other related schemes that did not measure the performance of their algorithms on any databases, we uses a database to evaluate the

Table 1. The performance of the proposed method by calculating the NC value and the retrieved logo under mentioned non geometric attacks in section 4.2

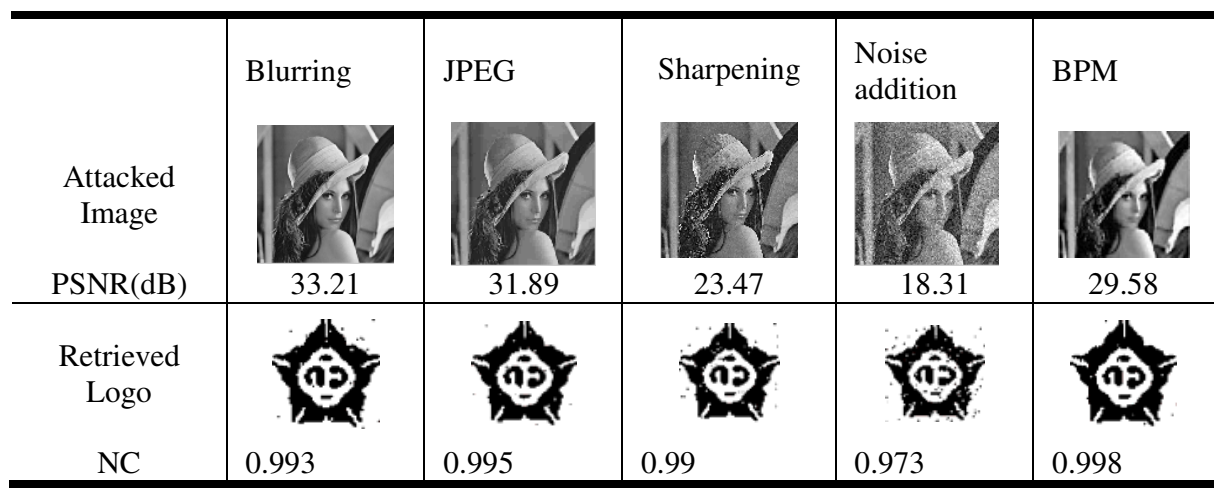


Table 2. The performance of the proposed method by calculating the NC value and the retrieved logo under mentioned geometric attacks in section 4.2

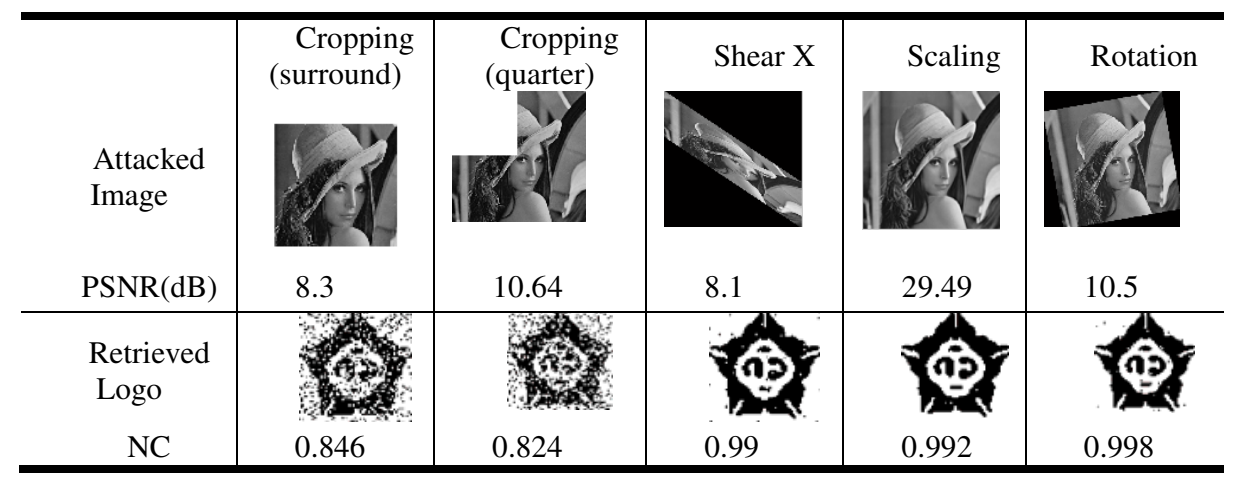

Table 3. Comparing NC between the proposed schemes in [4], [5], [9] and our method. The result is averaged over twelve randomly selected images with different complexities from Washington University database under the mentioned attacks in section 4.2.

\begin{tabular}{|c|c|c|c|c|c|}
\hline & Attack & $\begin{array}{c}\text { Scheme } \\
{[4]}\end{array}$ & $\begin{array}{c}\text { Scheme } \\
{[5]}\end{array}$ & $\begin{array}{c}\text { Scheme } \\
{[9]}\end{array}$ & $\begin{array}{c}\text { Proposed } \\
\text { scheme }\end{array}$ \\
\hline \multirow{6}{*}{ 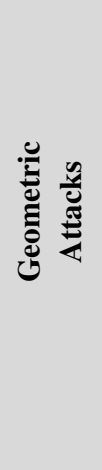 } & Rotation & 0.65 & 0.68 & 0.92 & 0.99 \\
\hline & $\begin{array}{c}\text { Shearing } \\
\text { in } \mathrm{x} \text { direction }\end{array}$ & 0.49 & 0.49 & 0.58 & 0.98 \\
\hline & $\begin{array}{c}\text { Shearing } \\
\text { in y direction } \\
\end{array}$ & 0.51 & 0.51 & 0.63 & 0.99 \\
\hline & $\begin{array}{l}\text { Surround } \\
\text { cropping }\end{array}$ & 0.53 & 0.51 & 0.72 & 0.83 \\
\hline & $\begin{array}{l}\text { Quarter } \\
\text { cropping }\end{array}$ & 0.73 & 0.72 & 0.99 & 0.78 \\
\hline & Scaling & 0.98 & 0.99 & 1 & 0.99 \\
\hline \multirow{5}{*}{ 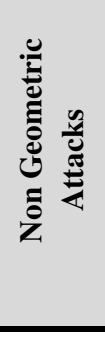 } & Blurring & 0.99 & 0.99 & 1 & 0.99 \\
\hline & Noising & 0.98 & 0.99 & 1 & 0.97 \\
\hline & JPEG & 0.99 & 0.99 & 1 & 0.99 \\
\hline & $\begin{array}{l}\text { Sharpen- } \\
\text { ing }\end{array}$ & 0.98 & 0.98 & 1 & 0.98 \\
\hline & ВРМ & 0.97 & 0.98 & 1 & 0.99 \\
\hline & Average & 0.80 & 0.80 & 0.89 & 0.95 \\
\hline
\end{tabular}


robustness of our proposed method. As the robustness of each scheme is dependent on the complexity of test image, we classified images of Washington University database [15] into low, medium and high complexity based on quad tree representation [14]. Then we randomly selected four images from each complexity as test images and we simulated our experiments on these 12 test images.

We applied the above-mentioned attacks on each 12 test images. Table 3 summarizes the average NC under above attacks over 12 test images in schemes [4], [5], [9] and our method. As indicated in Table 3, for geometric attacks our scheme outperforms other schemes in most cases such as rotation and shearing. For non geometric attacks although other schemes have better performance than ours, the differences in $\mathrm{NC}$ values are small. However, Table 3 shows that our scheme gives higher average $\mathrm{NC}$ in comparison with other schemes.

\section{Conclusion}

As zero-watermarking algorithms do not degrade the quality of the original images, it has become a new research area in recent years. These methods extract binary features from the host image and then apply exclusive-or between the binary features and a binary logo to generate a verification map. The resulted verification map is registered in a trusted authority for further protection. One of the most important issues in copyright protection schemes is how to retrieve the significant binary features from an image that is aimed to be watermarked. In this paper we used discrete wavelet transformation, cellular automaton and an effective normalization procedure to extract binary features from an image. The normalization procedure is used to provide robustness against geometric distortions of the image. Also, a cellular automaton is utilized to maintain the robustness against noises.

Experimental results demonstrate that the proposed scheme has high performance against common geometric attacks and sufficient robustness under non geometric attacks. Consequently, according to the simulation results on images with different complexities our proposed method outperforms other similar schemes [4], [5] and [9] in most cases.

\section{References}

1. Langelaar, G., Setyawan, I., Lagendijk, R.: Watermarking digital image and video data: A state-of-the-art overview. IEEE Signal Processing Magazine 17(5), 20-46 (2000)

2. Lee, W.B., Chen, T.H.: A public verifiable copy protection technique for still images. J. Syst. Softw. 62, 195-204 (2002)

3. Hsu, C.T., Wu, J.L.: Multiresolution watermarking for digital images. IEEE Trans. Circuits Syst. II, Analog Digit. Signal Process. 45(8), 1097-1101 (1998)

4. Chen, T.H., Horng, G., Lee, W.B.: A publicly verifiable copyright proving scheme resistant to malicious attacks. IEEE Transactions on Industrial Electronics 52(1), 327-334 (2005) 
5. Abdel-Wahab, M.A., Selim, H., Sayed, U.: A novel robust watermarking scheme for copyright-proving. In: The 2009 International Conference on Computer Engineering and Systems, ICCES 2009, art. no. 5383216, pp. 482-486 (2009)

6. Chang, C.C., Lin, P.Y.: Adaptive watermark mechanism for rightful ownership protection. The Journal of Systems and Software 81, 1118-1129 (2008)

7. Kazakova, N., Margala, M., Durdle, N.G.: Sobel edge detection processor for a real-time volume rendering system. In: Proceedings of the 2004 International Symposium on Circuits and Systems, vol. 2, pp. 913-916 (2004)

8. Lee, M.T., Chen, S.S.: Image Copyright Protection Scheme Using Sobel Technology and Genetic Algorithm. In: International Symposium on Computer, Communication, Control and Automation (2010)

9. Lin, W.H., Horng, S.J., Kao, T.W., Chen, R.J., Chen, Y.H.: Image copyright protection with forward error correction. Expert Systems with Applications 14(12) (2005)

10. Popovici, A., Popovici, D.: Cellular automata in image processing. In: Proceedings of 15th International Symposium on Mathematical Theory of Networks and Systems (2002)

11. Dong, P., Brankov, J.G., Galatsanos, N.P., Yang, Y., Davoine, F.: Digital Watermarking Robust to Geometric Distortions. IEEE Transaction on Image Processing 36, 11888-11894 (2009)

12. Rothe, I., Susse, H., Voss, K.: The method of normalization to determine invariants. IEEE Transaction on Pattern Analysis and Machine Intelligence 18(4), 366-376 (1996)

13. Hsu, C.T., Wu, J.L.: Hidden digital watermarks in images. IEEE Transaction on Image Processing 8(1), 58-68 (1999)

14. Yaghmaee, F., Jamzad, M.: Computing watermark capacity in images according to their quad tree. In: Int. IEEE 5th Symposium on Signal Processing and Information Technology, pp. 823-826. IEEE Press (2005)

15. Image data base,

http://www.cs.washington. edu/research/imagedatabase 